\title{
Effects of task-irrelevant texture motion on time-to-contact judgments
}

\author{
Daniel Oberfeld • Heiko Hecht • Klaus Landwehr
}

Published online: 16 November 2010

(C) Psychonomic Society, Inc. 2010

\begin{abstract}
We investigated the effect of local texture motion on time-to-contact (TTC) estimation. In Experiment 1, observers estimated the TTC of a looming disk with a spiral texture pattern in a prediction-motion task. Rotation of the spiral texture in a direction causing illusory contraction resulted in a significant TTC overestimation, relative to a condition without texture rotation. This would be consistent with an intrusion of task-irrelevant local upon task-relevant global information. However, illusory expansion did not cause a relative TTC underestimation but rather also a tendency towards overestimation. In Experiment 2, a vertical cylinder moved on the frontoparallel plane. Observers judged its TTC with a finish line. The cylinder was textured with stripes oriented in parallel to its longitudinal axis. It was either not rotating, rotating such that the stripes moved towards the finish line (i.e., in the same direction as the contour), or rotating such that the stripes moved away from the finish line. Both types of texture motion caused TTC overestimation compared to the static condition. Experiment 3 showed that the different effects of task-relevant and task-irrelevant texture motion are not a mere procedural effect of the prediction-motion task. In conclusion, task-irrelevant local motion and global motion are neither averaged in a simple manner nor are they processed independently.
\end{abstract}

Keywords Time-to-arrival · Time-to-contact $\cdot$ Visual perception - Texture - Motion - TTC theory - Contrast effect . Visual motion $\cdot$ Time-to-collision

D. Oberfeld $(\bowtie) \cdot$ H. Hecht $\cdot$ K. Landwehr

Department of Psychology, Johannes Gutenberg-Universität, D-55099 Mainz, Germany

e-mail: oberfeld@uni-mainz.de

\section{Introduction}

The time-to-contact (TTC), also called time-to-collision (Purdy, 1958) or time-to-arrival (Schiff \& Oldak, 1990), is the time it takes for a moving object to reach an observer or another point in space. According to Hoyle's (1957) analysis, for straight trajectories, constant velocities, and rigid non-rotating objects, TTC is optically specified by the ratio of a given, momentary visual angle and that angle's first temporal derivative. This optical variable has been termed $\tau$ (cf. Lee, 1976). In the case of head-on collision approaches, the outer contours of the object are typically considered to provide the relevant information about visual angle. However, in principle, any two points on the object's surface specify TTC just as well (cf. Bootsma \& Craig, 2002; Tresilian, 1990, 1991). The relevant angle whose relative rate of change specifies TTC could be based on various points within the object, or even on a patch of surface area in the case of a head-on collision. It is thus not a foregone conclusion that TTC judgments are based on the contours of the moving objects. In fact, it has been demonstrated that the sensitivity for various directions of visual motion increases with the number of visible display elements (Morrone, Burr, \& Vaina, 1995), although LópezMoliner, Brenner, and Smeets (2007) did not observe an increase in the accuracy of time-to-passage judgments when a surface texture was added to an approaching object.

We investigated cases where TTC is over-specified and judgments could be based on contour information and/or on texture expansion. In other cases, we pitted contour and texture motion against one another. We hypothesized that TTC estimates are not based on contour information alone but rather that surface texture motion plays an important role.

During the last four decades, considerable effort has been devoted to the question whether humans and other 
species use the optical variable $\tau$ or other sources of information for TTC judgments (see Hecht \& Savelsbergh, 2004 for a recent overview of data and theories). A simplifying summary of these results is that observers are able to base their judgments on $\tau$ but often resort to other optical variables if these provide information about TTC (e.g., DeLucia, Kaiser, Bush, Meyer, \& Sweet, 2003; Kerzel, Hecht, \& Kim, 1999). If we thus take seriously the possibility that the human visual system may make flexible use of the available optical variables, a closer investigation of the nature of the information being extracted from optic flow becomes necessary. A number of studies have looked at relative contributions of components of optic flow that may guide TTC estimation. For instance, Beverley and Regan (1983) introduced a mismatch paradigm to dissociate effects of changing coarseness of local surface texture and changes in the overall size of an object. A randomly chequered 2D square was simulated to either approach, or recede from, a stationary observer. The texture was manipulated such that it was magnified in synchrony with, slower than, or faster than the square's contours. In the former case, a stronger motion-indepth aftereffect was elicited than for the remaining conditions. Vincent and Regan (1997) adopted this paradigm for TTC research and found responses to be accurate in the consistent, synchronized condition only. Their participants viewed a looming square approaching on a direct collision course. The square was textured with a random checkerboard-like pattern. If the rate of expansion of the texture elements was smaller than the rate of expansion of the contour (the square), then the observers overestimated TTC. This effect was strongest if the texture expansion rate was zero, that is, when the texture elements remained fixed in size across the trial duration. If the texture elements expanded faster than the contour, then TTC was underestimated. Thus, participants behaved as if they had used a weighted average of TTC information from global and local motion. Subsequent experiments showed that the specific make-up of the stimulus display is critical. Gray and Regan (1999) used dot stimuli and varied the size and the separation of texture elements independently. Dots either magnified in accordance with separation, or they remained fixed in size. In the latter condition, substantial overestimation of TTC was found when fixed dot size exceeded 4.4 arc min. Harris and Giachritsis (2000) let individual dots or small clusters of non-expanding dots expand, either faster or slower than the whole array of display elements. They found effects of the ratio between local expansion rate and global expansion rate comparable to the results of Vincent and Regan (1997).

In our opinion, there is a simple explanation for the above findings. In our natural environment, the TTC information provided by the expansion of the contour and by the expansion of the texture elements of an approaching rigid object is typically highly consistent, although not necessarily identical, as we will discuss below. Thus, both the global and the local visual motion are normally task-relevant because both types of information specify an object's TTC.

The inconsistent, mismatched stimuli used, for example, by Vincent and Regan (1997) optically specify something different from a plain approach. For instance, if the texture expansion rate is much smaller than the expansion rate of the contour, then the motion of the contour can be perceived as a moving aperture rather than as an approaching object, as noted by Beverley and Regan (1983, p. 1388) and Vincent and Regan (1997, p. 36, footnote 3). And constant-size dots or texture elements signal an infinite TTC (Gray \& Regan, 1999). Thus, the observed effects could be caused by the "impossible" nature of the stimuli rather than being indicative of a general averaging of local and global TTC information. We propose that convincing evidence for such a cross-talk would have to be derived from ecologically plausible stimuli. We suggest that distractor displays can furnish such stimuli. Here, a virtual object would be simulated, and some surface property would create a potential distraction. We know that the mere presence of a second irrelevant object, which also produces an expanding optic flow, can bias the TTC judgements of the relevant approaching object. Oberfeld and Hecht (2008) presented a target object (for which a TTC estimate was required within a prediction motion paradigm) together with an additional moving distractor object that provided no information concerning the target's TTC and thus was entirely task-irrelevant. Observers were unable to ignore the TTC information provided by the distractor. Surprisingly, the data showed no evidence for information integration. Instead, regardless of whether the distractor TTC was earlier or later than the target TTC, the presence of the distractor resulted in a relative underestimation of target TTC. A tentative explanation is that observers may have used a safety strategy, in the sense that the additional approaching object was identified as a potential threat, so that it made observers more cautious when they considered an action. Be this as it may, the integration of TTC information from different sources in physically possible stimuli has yet to be demonstrated. We designed several experiments to pursue this venue.

Before turning to a description of the experiments, we want to come back to the issue of to which extent the contour expansion and the texture expansion of nonlaboratory objects provide consistent information about TTC. For a rigid object, the TTC signaled by a $\tau$-type optical variable based on the visual angle subtended by the contour is very similar to alternative "local $\tau$ 's" (Tresilian, 1991), but not in all cases identical. As an example, imagine a soccer ball (i.e., a sphere) textured with the typical black-and-white pattern, approaching the observer 
on a direct collision course. Now $\tau$ for the central texture element centered on the line of sight will be slightly lower than for another texture element of identical size but positioned on the right side of the ball, and thus farther away from the observer (López-Moliner, et al., 2007). Still, time-to-passage information (Kaiser \& Mowafy, 1993) provided by the angle between one point on the distant surface patch and the line of sight would be close to the contour $\tau$. What if this soccer ball rotates? Then, the texture elements on one hemisphere will have a higher expansion rate than the contour, but the texture elements on the other hemisphere will have a smaller expansion rate. Thus, this texture motion would still differ from laboratory stimuli showing a homogeneous mismatch between global and local expansion rate (Vincent \& Regan, 1997) ${ }^{1}$. Could nonrigid objects show such a pattern? For an inflating or deflating balloon (cf. Savelsbergh, Whiting, \& Bootsma, 1991), the expansion rates of the contour and of the texture are correlated. Compared to a balloon remaining constant in size, a deflating balloon will exhibit a smaller global, but also a smaller local, expansion rate. Thus, the two rates will still provide roughly consistent information.

Considering more extreme cases, one might even argue that it is physically impossible that an object shows global (contour) expansion but at the same time local (texture) contraction. However, a chemical process acting on the surface of an object and creating a time-varying texture might in principle produce such a situation. The important point we wish to make, however, is that objects showing a strong mismatch between contour expansion and texture expansion are difficult to imagine, simply because in our natural environment they are rare. Thus, even considering the examples discussed above, it can be concluded that stimuli showing such a strong mismatch are not ecologically plausible. In contrast, in the experiments presented in this paper, we used stimuli corresponding to an ecologically plausible approach of a rigid object. ${ }^{2}$

The present study was aimed at answering the question of how task-irrelevant texture motion may influence TTC judgments, if at all. In our first experiment, observers

\footnotetext{
${ }^{1}$ Note that rotating nonspherical objects can produce at least two different and potentially inconsistent contour expansion rates. Kaiser and Hecht (1994) presented rotating brick-shaped objects that were on a collision course towards the stationary observer. The objects either rotated such that the broad side faced the observer initially and the narrow side faced her just before disappearance, or the objects rotated in the opposite manner. It turned out that observers could compensate for simple target rotations (i.e., around a single axis), but not so for complex rotations. In these complex rotations, the TTC estimates reflected a weighted average between the two rates of contour expansion. A similar effect was reported by Gray and Regan (2000) using essentially the same experimental manipulations with a nonspherical object shaped like a rugby ball.

${ }^{2}$ This notwithstanding, the stimuli were of the laboratory type and thus not genuinely ecological.
}

viewed a disk with a spiral texture pattern approaching them on a direct collision course. Such an object is physically possible as all parts were simulated to expand as a function of distance from the observer's eye-point. The disk was either non-rotating, or rotating such that there was either illusory expansion or illusory contraction (cf. Holland, 1965; Plateau, 1850). The rotation of the texture pattern was task-irrelevant because (1) it provided no information regarding the TTC of the object, and (2) each condition corresponded to an ecologically plausible approach of a rigid object. There are at least three different potential outcomes of this experiment: (1) observers might be successful in ignoring the task-irrelevant information so that the texture rotation would have no effect on the TTC estimates; (2) observers might indeed use a weighted average between the expansion of the contour and the illusory expansion/contraction of the texture motion, as suggested by Vincent and Regan (1997); or (3) the taskirrelevant texture motion could cause a general over- or underestimation of TTC akin to the effects of a taskirrelevant distractor object (Oberfeld \& Hecht, 2008).

Experiment 2 was designed to assess converging evidence from translatory motion in the frontoparallel plane. If local texture generally interferes with global TTC information, then an expanding flow-field should not be a prerequisite. Lateral motion creates a $\tau$-variable without any local expansion. Thus, the potential conflict between constant size dots versus expanding spaces between them is avoided. Observers judged a cylinder moving from left to right at constant speed. At varying distances from the finish line, the cylinder was removed from view and its arrival time was estimated by the observer. The cylinder textured with stripes parallel to its longitudinal symmetry axis either rotated such that the visible stripes moved towards the finish line (i.e. consistent with a rolling object), or rotated in the reverse direction (i.e., in the opposite direction as the contour). Finally, Experiment 3 was designed to test whether the difference between the results from Experiments 1 and 2 and previous "mismatch" studies (e.g., Vincent \& Regan, 1997) could be attributed to the use of different psychophysical procedures.

\section{Experiment 1: a looming spiral}

Observers viewed a disk in virtual reality approaching them on the mid-saggital plane. At time $t_{0}$, the disk started to move at constant speed (see Fig. 1a). At time $t_{\text {blank }}$, the object disappeared from the screen. A prediction-motion (PM) task was used (Schiff \& Detwiler, 1979). The observers were asked to press a button at exactly the instant when they thought the object would have arrived at their position had it continued its trajectory. We designate by 
a

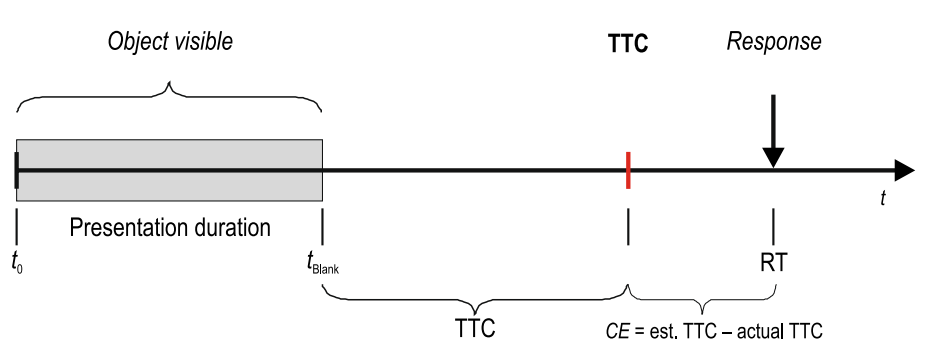

b

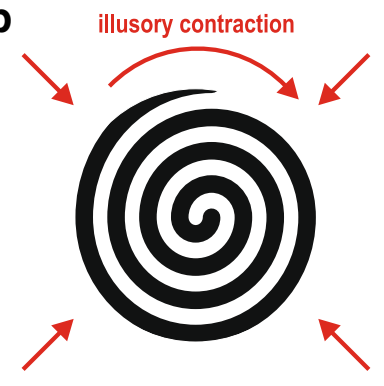

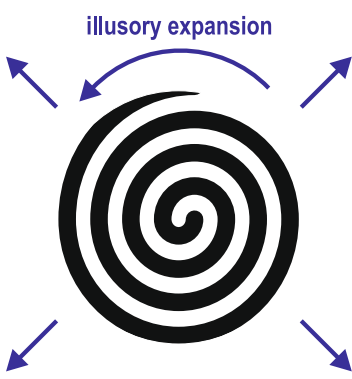

Fig. 1 Experiment 1. a Temporal structure of a trial (see text). b The spiral texture rotated, eliciting either the illusory perception of expansion, or of contraction. In the static condition, the texture did not rotate

TTC the time between the object's disappearance from the screen and the instant at which the object would have reached the observer. Heuer (1993) termed this time interval "final time-to-contact" in order to distinguish it from the time between the object's appearance on the screen and the instant at which the object would have reached the observer.

To test for an effect of task-irrelevant texture motion, we presented a disk textured with an Archimedean spiral (cf. Scott \& Noland, 1965). Rotating this spiral in its winding direction elicits the illusion of contraction (see Fig. 1b). Rotating it in the reverse direction causes illusory expansion. If observers were to integrate the global and the local motion signals during the estimation process, then, relative to the static condition without rotation, the expanding texture should result in shorter TTC estimates (i.e., relative underestimation), whereas the contracting texture should result in longer TTC estimates (i.e., relative overestimation).

\section{Method}

\section{Observers}

Twenty volunteers (13 women, 7 men) participated in the experiment for partial course credit or payment $(€ 5 / \mathrm{h})$. The participants ranged in age from 19 to 39 years (mean 24.0 years, SD 6.0). All had normal or corrected-to-normal vision. Once the topic of the study and potential risks had been explained to them, all participants gave written informed consent according to the Declaration of Helsinki. They were uninformed about the experimental hypotheses.

\section{Stimuli and apparatus}

The stimuli were created using the animation software Vizard (WorldViz LLC, Santa Barbara, USA) and presented on a CRT display (Samtron 98PDF, active display size $312 \mathrm{~mm} \times$ $234 \mathrm{~mm}$ horizontal $\times$ vertical, refresh rate $70 \mathrm{~Hz}$ noninterlaced, horizontal dot pitch $0.2 \mathrm{~mm}$ ). The animation update rate was approximately 60 frames/s. The display had a resolution of 1,280 × 1,024 pixels (horizontal by vertical). A
Dell Precision 650 computer equipped with an NVIDIA Quadro4 900 XGL graphics adaptor was used for animation and experimental control.

The observers were tested individually in a dimly lit room watching the CRT display binocularly from a distance of $40 \mathrm{~cm}^{3}$ The display subtended $42.6^{\circ}$ of visual angle horizontally and $32.6^{\circ}$ vertically. A chinrest was used to align the line of sight with the center of the display.

The stimulus was a rigid disk moving towards the observer at constant speed on a collision course. The disk was horizontally centered on the screen and its surface was oriented orthogonally to the line of sight. For an observer fixating the midpoint of the object, the object moved on the mid-saggital plane. A black-and-white spiral texture with two possible winding directions was mapped onto the disk. Figure $1 \mathrm{~b}$ shows the version with clockwise winding direction. The background was uniformly black. The simulation corresponded to a disk with a diameter of $4 \mathrm{~m}$ starting at distances beyond $20 \mathrm{~m}$ from the observer. Across conditions (see below), the disk subtended a visual angle between 3.9 and $21.8^{\circ}$ at the instant before it disappeared.

\section{Procedure and design}

Trials showing different approaches of the object were created by varying the presentation duration, the TTC, and the velocity of the disk. The presentation duration was $t_{\text {blank }}=$ $500,1,250$, or $2,000 \mathrm{~ms}$. We varied this parameter for two reasons. First, it is conceivable that the texture motion has an effect only if it can be perceived for a sufficient time, which would suggest the involvement of slower, maybe cognitive processes (e.g., Tresilian, 1995). Second, varying the

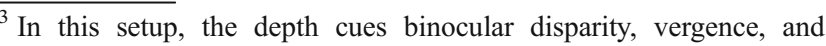
accommodation signaled a rather small and constant depth, and thus implicated an infinite TTC. As discussed by Oberfeld and Hecht (2008, p. 614), this might cause a tendency towards TTC overestimation. However, this conflict was equally present for all experimental conditions. Therefore, the effects of the different types of texture motion on the TTC estimates found in the experiments reported here cannot be attributed to the display setup.
} 
presentation duration reduces the correlation between optical variables like the initial and final object size and TTC (cf. Gray \& Regan, 1998; Oberfeld \& Hecht, 2008). The TTC was $500,1,000,1,500$, or $2,000 \mathrm{~ms}$. Disk velocity $v$ was varied between 20.8 and $29.2 \mathrm{~m} / \mathrm{s}$ in $1.4 \mathrm{~m} / \mathrm{s}$ steps. For each combination of velocity and presentation duration, a starting distance was computed to produce the designated TTC, resulting in starting distances between 20.8 and $116.8 \mathrm{~m}$.

The experimental variable of primary interest was texture motion. Each of the two spiral textures was either presented rotating with $360 \%$ s creating an impression of expansion, or rotating with $360 \%$ in the opposite direction creating the impression of contraction (Plateau, 1850; Scott \& Noland, 1965). In the static condition, the texture did not rotate.

The observer pressed a response button with the index finger of the dominant hand at the instant at which he or she thought that the object would have arrived at his or her position, had it continued its trajectory. The observer then initiated the next trial by pressing another designated button on the response pad.

The experiment started with a practice block containing only trials from the static condition (i.e., without texture rotation). From this condition, all trials with the slowest, intermediate, and highest velocity were presented (72 trials). Visual trial-by-trial feedback was provided in form of the signed deviation in milliseconds of the time of the observer's key-press from the actual target TTC. Observers were informed that the initial and final size of the object, its speed, and the presentation duration would be varied and that none of these variables provided a reliable cue to contact time.

In the main part of the experiment no feedback was provided. Each observer received each of the 3 (presentation duration) $\times 4($ TTC $) \times 7$ (velocity) $\times 3$ (texture motion) $\times$ 2 (winding direction) combinations once, resulting in a total of 504 trials. Presentation order was randomized. The experimental session lasted approximately $65 \mathrm{~min}$, including two short breaks.

\section{Results and discussion}

The individual data were analyzed in terms of constant error (CE; cf. Hartmann, 1983; Tresilian, 1994). Constant error denotes the signed difference between the observed response time RT (i.e., the time between the disappearance of the target object and the key-press) and the correct response time (i.e., the designated TTC; see Fig. 1a). A repeated-measures ANOVA using a univariate approach was conducted on CE. The Huynh-Feldt correction for the degrees-of-freedom $(d f)$ was used (Huynh \& Feldt, 1976) and the value of the $d f$ correction factor $\widetilde{\varepsilon}$ is reported. Partial $\eta^{2}$ is reported as a measure of association strength. An $\alpha$-level of .05 was used for all analyses reported in this paper and all reported $p$-values are two-tailed. In the ANOVA, the within-subjects factors were texture motion, winding direction, presentation duration, TTC, and velocity. The ANOVA results are displayed in Table 1. There was a significant main effect of texture motion on CE. As can be seen in Fig. 2, the object with the contracting spiral was judged on average to arrive $176 \mathrm{~ms}$ later than the object with the static texture. Post-hoc pairwise comparisons between all pairs of texture motion were computed using non-pooled error terms (i.e., by computing separate pairedsamples $t$ tests; Keselman, 1994) and Hochberg's (1988) sequentially acceptive step-up Bonferroni procedure, which controls the familywise Type I error rate. We used an alpha-level of .05. These tests indicated a significant difference between the $\mathrm{CE}$ obtained with the contracting and the static texture motion. The expanding spiral did not result in the expected underestimation of TTC relative to the static condition, but descriptively also in an overestimation (Fig. 2). However, this difference in CE was not significant. Finally, the CEs observed with the expanding and the contracting texture differed significantly.

The ANOVA (Table 1) showed a significant Texture Motion $\times$ Presentation Duration interaction. As can be seen in Fig. 3a, at the shortest presentation duration, the contracting texture resulted in overestimation and the expanding texture resulted in underestimation of TTC, relative to the static condition. At the longer presentation durations, however, the expanding texture resulted in a relative overestimation. To better understand the Texture Motion $\times$ Presentation Duration interaction, we computed two post-hoc five factorial ANOVAs, comparing the
Table 1 Experiment 1: results of the repeated-measures ANOVA on the constant error. All significant effects, and the nonsignificant effects mentioned in the text are displayed

Bold font indicates significant effects. The value $\widetilde{\varepsilon}$ is the Huynh-Feldt correction factor for the degrees of freedom.

\begin{tabular}{lllllll}
\hline Factor & Num. $d f$ & Den. $d f$ & $F$ & $p$ & $\widetilde{\varepsilon}$ & Partial $\eta^{2}$ \\
\hline Texture motion $($ TM) & $\mathbf{2}$ & $\mathbf{3 8}$ & $\mathbf{3 1 . 4 4}$ & $<.001$ & $\mathbf{1 . 0 0}$ & $\mathbf{. 6 2 3}$ \\
Presentation duration $\left(t_{\text {blank }}\right)$ & 2 & 38 & 3.74 & .060 & .59 & .164 \\
TTC & $\mathbf{3}$ & $\mathbf{5 7}$ & $\mathbf{1 0 . 8 2}$ & $\mathbf{. 0 0 2}$ & $\mathbf{. 3 8}$ & $\mathbf{. 3 6 3}$ \\
Velocity $(v)$ & $\mathbf{6}$ & $\mathbf{1 1 4}$ & $\mathbf{3 0 . 0 0}$ & $<.001$ & $\mathbf{. 9 5}$ & $\mathbf{. 6 1 2}$ \\
TM $\times \boldsymbol{t}_{\text {blank }}$ & $\mathbf{4}$ & $\mathbf{7 6}$ & $\mathbf{7 . 1 4}$ & $<.001$ & $\mathbf{1 . 0 0}$ & $\mathbf{. 2 3 7}$ \\
TM $\times$ TTC & $\mathbf{6}$ & $\mathbf{1 1 4}$ & $\mathbf{2 . 6 4}$ & $\mathbf{. 0 2 2}$ & $\mathbf{. 6 7}$ & $\mathbf{. 1 2 2}$ \\
$\boldsymbol{t}_{\text {blank }} \times$ TTC & $\mathbf{6}$ & $\mathbf{1 1 4}$ & $\mathbf{4 . 9 8}$ & $\mathbf{. 0 0 2}$ & $\mathbf{. 5 9}$ & $\mathbf{. 2 0 8}$ \\
\hline
\end{tabular}




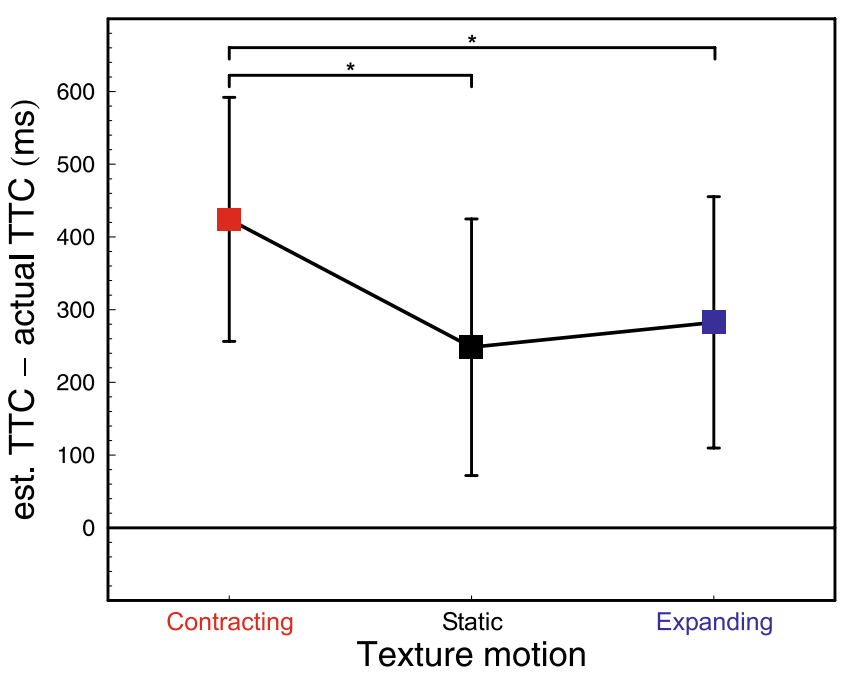

Fig. 2 Experiment 1. Mean constant error (i.e., the estimated TTC minus the actual TTC) as a function of texture motion. The brackets indicate significant pairwise differences ( $p<.05$, two-tailed). Error bars $95 \%$ confidence intervals (CIs)

expanding and the static texture, and the contracting and the static texture. Both showed a significant Texture Motion $\times$ Presentation Duration interaction, $F(2,38)=12.2, p<.001$, $\widetilde{\varepsilon}=1.0, \eta^{2}=.39$, and $F(2,38)=7.1, p<.001, \widetilde{\varepsilon}=1.0, \eta^{2}=$ .27 , respectively. Post-hoc pairwise comparisons between all pairs of levels of texture motion at a given presentation duration were computed, again using the method by Hochberg (1988). For the total of $3 \times 3=9$ pairs tested, only two tests were not significant at an alpha-level of .05 (expanding versus static at presentation durations $500 \mathrm{~ms}$ or $1,250 \mathrm{~ms})$.

In the main ANOVA (Table 1), there was also a significant Texture Motion $\times$ TTC interaction. As Fig. $3 b$ shows, the

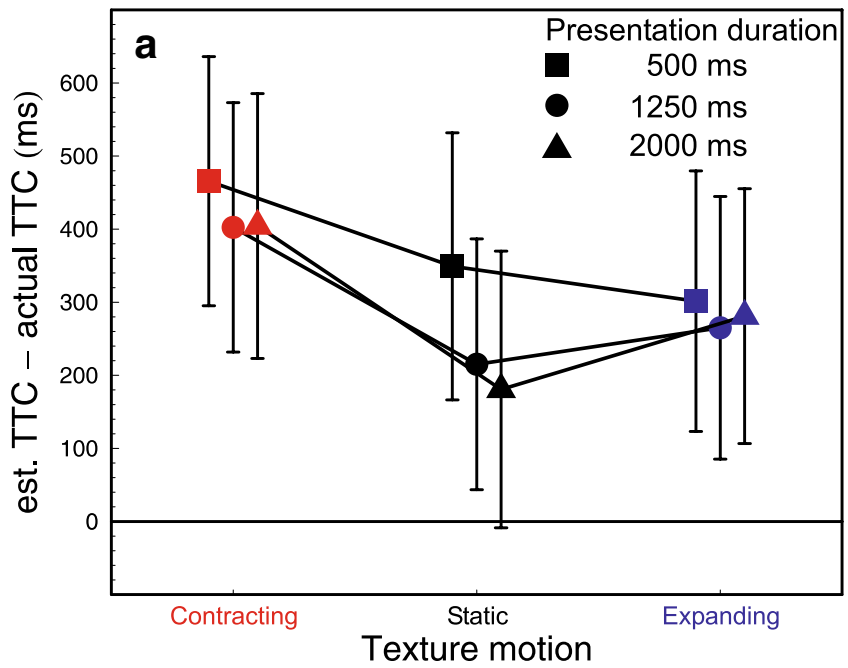

Fig. 3 Experiment 1. a Mean constant error as a function of texture motion and presentation duration. Boxes, circles, and triangles correspond to presentation durations of $500,1,250$, and $2,000 \mathrm{~ms}$, respectively. $\mathbf{b}$ Mean constant error as a function of texture motion and difference between the errors in the contracting and the static condition were smaller at the shortest than at the longer TTCs. In fact, with the data for TTC $=500 \mathrm{~ms}$ excluded, a post-hoc five-factorial ANOVA showed no significant Texture Motion $\times$ TTC interaction, $F(4,76)=0.68$.

In the original ANOVA (Table 1), none of the remaining interactions involving texture motion were significant at the .05-level.

In addition to the above effects of texture motion, the ANOVA reported in Table 1 indicated several significant effects of the motion parameters. As seen by the confidence intervals in Fig. 4a, the observers significantly overestimated TTC, except at the longest designated TTC. This result is compatible with previous studies where overestimation was observed at TTCs smaller than approximately 1,500 ms (Hecht, Kaiser, Savelsbergh, \& van der Kamp, 2002; Heuer, 1993; Oberfeld \& Hecht, 2008), while underestimation was found at longer TTCs (McLeod \& Ross, 1983; Schiff \& Detwiler, 1979). In the present experiment, the TTC overestimation decreased as the designated TTC increased from 1,000 to $2,000 \mathrm{~ms}$, as confirmed by a significant effect of TTC. Surprisingly, the overestimation was less pronounced at TTC $=500 \mathrm{~ms}$ than at $\mathrm{TTC}=1,000 \mathrm{~ms}$. The TTC $\times$ Presentation duration interaction was also significant, presumably due to the fact that the presentation duration had no effect at the shortest TTC (see Fig. 4a). The overestimation tended to be higher at the shortest presentation duration, but this effect failed to reach significance $(p=.060)$. As Fig. $4 \mathrm{~b}$ shows there was a significant effect of velocity. The amount of TTC overestimation increased with increasing velocity. There are two potential explanations for this effect. First, the observers may have placed a higher weight on the simple pictorial cue

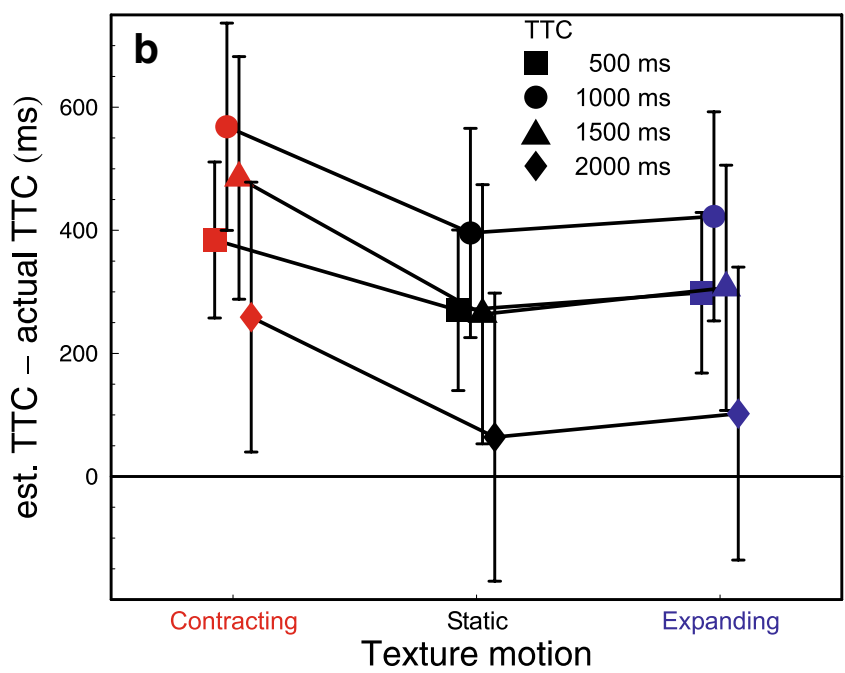

TTC. Boxes, circles, triangles, and diamonds correspond to TTCs of $500,1,000,1,500$, and 2,000 ms, respectively. Lines are shifted on the $x$-axis to reduce overlap. Error bars $95 \%$ CIs 


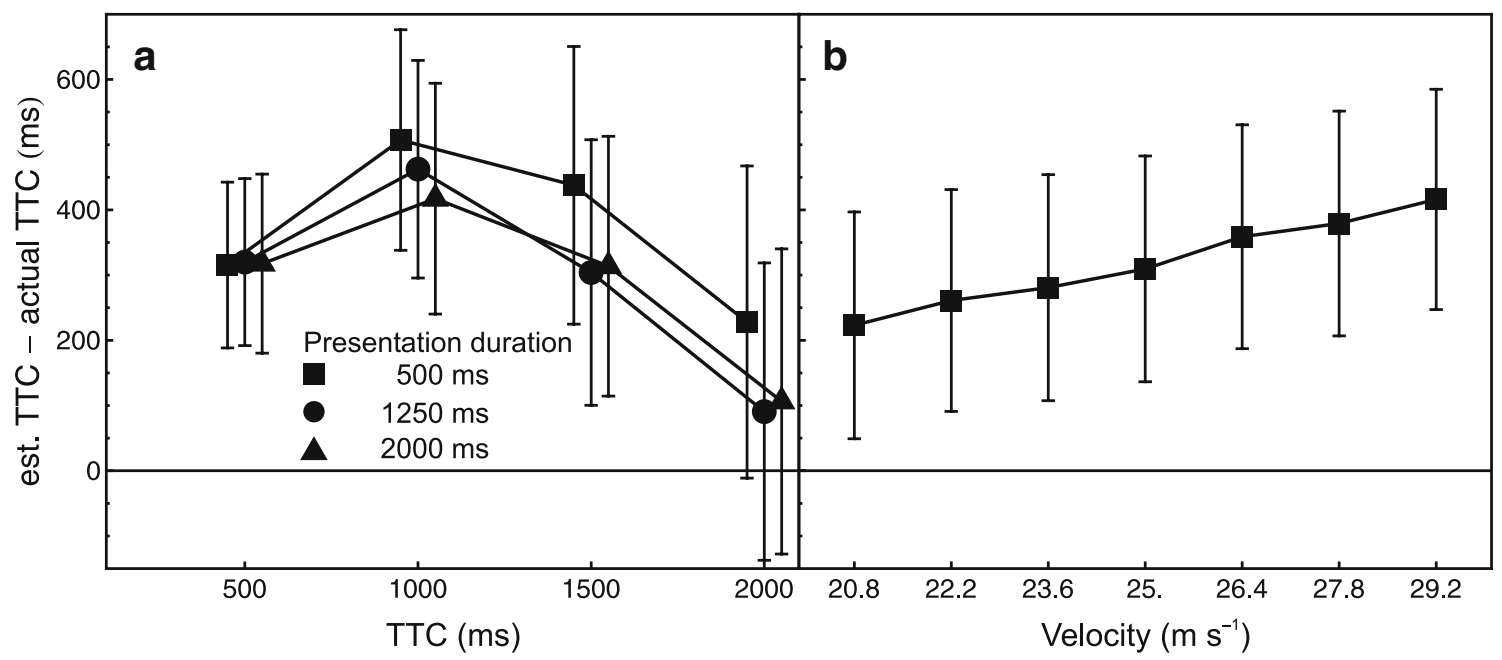

Fig. 4 Experiment 1. a Mean constant error as a function of TTC and presentation duration. Boxes, circles, and triangles correspond to presentation durations of $500,1,250$, and 2,000 ms, respectively. b Mean constant error as a function of velocity. Error bars $95 \%$ CIs

"final size" (DeLucia, 1991) than on the motion of the object (i.e., the velocity). For stimuli of equal final size, such a strategy would result in a relative underestimation of TTC at slow velocities, and a relative overestimation at fast velocities. Above that, due to the construction of the stimuli there was a weak negative correlation between the velocity and the final size of the object. As a consequence, responding on the basis of final size (that is, producing a small TTC estimate if the object was large at the instant where it disappeared from the screen) would again have resulted in smaller TTC estimates at the slow velocities than at the high velocities. The remaining effects were not significant (all $p$ values $>.05$ ). Taken together, the effects of the motion parameters follow the pattern typically observed in PM experiments.

Concerning the effects of task-irrelevant texture motion, we found evidence against the integration of global and local motion in the sense of an averaging process. While the contracting texture resulted in overestimation relative to the static condition, the expanding texture did not cause a significant relative underestimation of TTC. This indicates that the effects of task-irrelevant texture motion in an ecologically plausible setting differ from the effects of taskrelevant "impossible" texture motion. Only in the latter case were the data compatible with an averaging of global and local motion information (Giachritsis \& Harris, 2005; Harris \& Giachritsis, 2000; Vincent \& Regan, 1997). We also found that the effects of texture motion increased with increases in presentation duration and TTC. The effect of the latter parameter might be indicative of cognitive influences.

Could the effects of texture motion we observed be due to the use of specific strategies when estimating TTC? If, for instance, an observer tracked the angle between two fixed points on the spiral (e.g., the inner end of the spiral, and the outer end of the spiral), despite the movement of these points due to rotation, then $\tau$ information provided by the expansion of this angle would signal a TTC similar but not necessarily exactly identical to the TTC information provided by the expansion of the contour. The important point is, however, that this local $\tau$ would be independent of the direction of rotation. What if, alternatively, observers were able to track, for example, the lowest black point on the spiral (on an imaginary vertical line passing through the center of the disk), following its movement during rotation? In this case, the angle between this point and, for example, the center of the disk would vary due to the rotation of the spiral. More specifically, the tracked point moves inwards if there is illusory contraction and moves outwards if there is illusory expansion. As a consequence, using local $\tau$ between the described two points should result in a relative TTC overestimation for illusory contraction, and a relative underestimation for illusory expansion. On a more general level, if the disk with the rotating spiral was processed as a regularly expanding image as assumed by Lee (1974), then the texture motion should have no effect whatsoever on the TTC estimate. If, however, the TTC estimation process uses illusory motion, then the illusory expansion should produce shorter TTC estimates than a disk without rotation. None of the alternative patterns discussed above is compatible with our data.

As a final note, the results of Experiment 1 demonstrate that dynamic illusion displays affect judgments of time-tocontact, just as static illusion displays have been shown to do (DeLucia, Tresilian, \& Meyer, 2000).

\section{Experiment 2: motion in the frontoparallel plane}

Experiment 2 was conducted to test whether the results from Experiment 1 generalize to other types of task- 
irrelevant texture motion, or represent a rather specific effect of the rotating spiral textures presented in Experiment 1. Equally important, we were interested in whether the effects generalize to TTC judgments for motion in the frontoparallel plane.

\section{Method}

\section{Observers}

Twenty volunteers (13 women, 7 men) participated in the experiment for partial course credit or payment $(€ 5 / \mathrm{h})$. The participants ranged in age from 19 to 58 years (mean 26.0, SD 10.1). As before, all had normal or corrected-to-normal vision and gave written informed consent according to the Declaration of Helsinki.

\section{Stimuli and apparatus}

The same apparatus as in Experiment 1 was used.

The stimulus was a cylinder with its longitudinal symmetry axis oriented vertically and moving at constant speed towards a yellow finish line near the right edge of the display (see Fig. 5). A black-and-white stripes texture was mapped onto the cylinder. The cylinder was rendered threedimensionally with the animation software Vizard. The observers viewed a two-dimensional projection of the $3 \mathrm{D}$ object. The object had a screen size of $26 \mathrm{~mm}$ horizontally and $31 \mathrm{~mm}$ vertically. The width of the black and white stripes was identical (horizontal screen size $4 \mathrm{~mm}$ for a stripe centered horizontally at a virtual line orthogonal to the screen surface).

The finish line had a thickness of $3 \mathrm{~mm}$ and was positioned $30 \mathrm{~mm}$ from the right edge of the screen. The observers viewed the display binocularly from a distance of

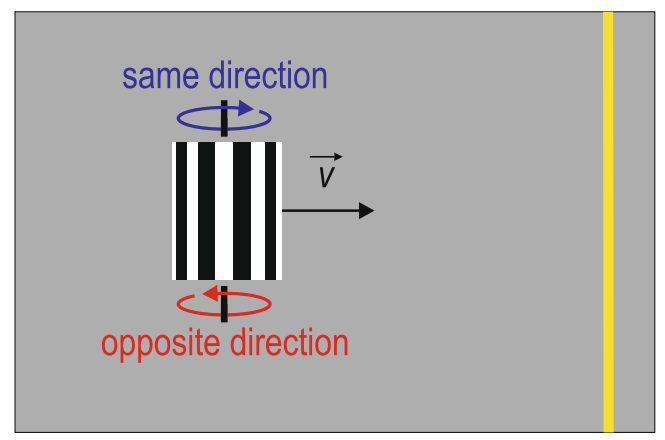

Fig. 5 Experiment 2. Schematic depiction of the stimuli. A cylinder with a black-and-white stripes texture approached a finish line located on the right edge of the display at constant speed $v$. The cylinder rotated about its longitudinal symmetry axis (symbolized by the vertical lines) so that the visible stripes either moved in the same direction as the object, or moved in the opposite direction. In the static condition, the cylinder did not rotate
$40 \mathrm{~cm}$, using the chin rest that aligned their line of sight with the center of the screen.

When its position was vertically and horizontally centered on the screen the object subtended a visual angle of $3.7^{\circ}$ horizontally and $4.4^{\circ}$ vertically, and the stripe closest to the observer subtended a visual angle of $0.57^{\circ}$ horizontally.

The background was uniformly black.

\section{Procedure and design}

Trials showing different approaches of the object moving from left to right towards the finish line were created by varying the presentation duration, the TTC, and the velocity. The presentation duration was $500,1,250$, or $2,000 \mathrm{~ms}$. The TTC was $500,1,000,1,500$, or $2,000 \mathrm{~ms}$. The velocity was varied between 59 and $64 \mathrm{~mm} / \mathrm{s}$ in six steps. Such a relatively narrow range of velocities was necessary due to the limited display size. For each combination of velocity and presentation duration, a starting distance (i.e., distance in $\mathrm{mm}$ from the right edge of the cylinder to the left edge of the finish line) was computed to produce the designated TTC, resulting in starting distances between 59 and $256 \mathrm{~mm}$.

The critical variable was again the texture motion. The cylinder was presented without rotation (static), with a rotation about its longitudinal symmetry axis $\left(180^{\circ} / \mathrm{s}\right)$ so that the stripes on the part of the cylinder facing the observer moved in the same direction as the object, or with a rotation $\left(180^{\circ} / \mathrm{s}\right)$ in the reverse direction so that the stripes moved in the opposite direction as the object. If the participants used a weighted average of the global (contour) and the local (texture) motion signals, then the same-direction texture motion should result in TTC underestimation relative to the static condition, whereas the opposite-direction texture motion should result in relative overestimation.

The observer pressed a response button with the index finger of the dominant hand at the instant when he or she thought that the cylinder would first touch the finish line. The observer then initiated the next trial by pressing another designated button on the response pad.

The experiment started with a practice block containing only trials from the static condition (i.e., without rotation of the cylinder). From this condition, all trials with the slowest, intermediate, and highest velocity were presented (36 trials). Visual trial-by-trial feedback was provided in form of the signed TTC error. The observers were informed that the initial position of the object, the final position, the speed, and the presentation duration would be varied and that none of these variables provided a reliable cue to contact time.

In the main part of the experiment, no feedback was provided. Each observer received each of the 3 (presenta- 
tion duration) $\times 4($ TTC) $\times 7$ (velocity) $\times 3$ (texture motion) combinations once, resulting in a total of 252 trials. Presentation order was randomized. The experimental session lasted approximately $40 \mathrm{~min}$, including two short breaks.

\section{Results and discussion}

To analyze the effects of the texture motion on the CE, a repeated-measures ANOVA with Huynh-Feldt correction for the degrees-of-freedom was conducted. The within-subjects factors were texture motion, presentation duration, TTC, and velocity. There was a significant main effect of texture motion on the $\operatorname{CE}, F(2,38)=39.4, p<.001, \widetilde{\varepsilon}=1.0, \eta^{2}=.68$. As can be seen in Fig. 6, the object with texture motion opposite to its global motion was judged to arrive $173 \mathrm{~ms}$ later than the object with the static texture. This is compatible with the hypothesis that texture motion in the opposite direction is averaged into the global TTC estimate and thus causes its overestimation. However, texture motion in the same direction as the contour motion did not result in a relative underestimation, but rather in a slight overestimation, akin to the effect of the expanding texture in Experiment 1. Post-hoc pairwise comparisons showed a significant difference between the opposite-direction and the static condition, $t(19)=$ 8.31, $p<.001$, as well as the difference between the opposite-direction and the same-direction condition, $t(19)=$ 7.13, $p<.001$. The difference between the same-direction condition and the static condition just failed to reach significance, $t(19)=2.09, p=0.0504$.

In the ANOVA, the Texture Motion $\times$ TTC interaction was significant, $F(6,114)=4.70, p<.001, \widetilde{\varepsilon}=.79, \eta^{2}=.20$. As Fig. 7a shows, the effect of texture motion increased with

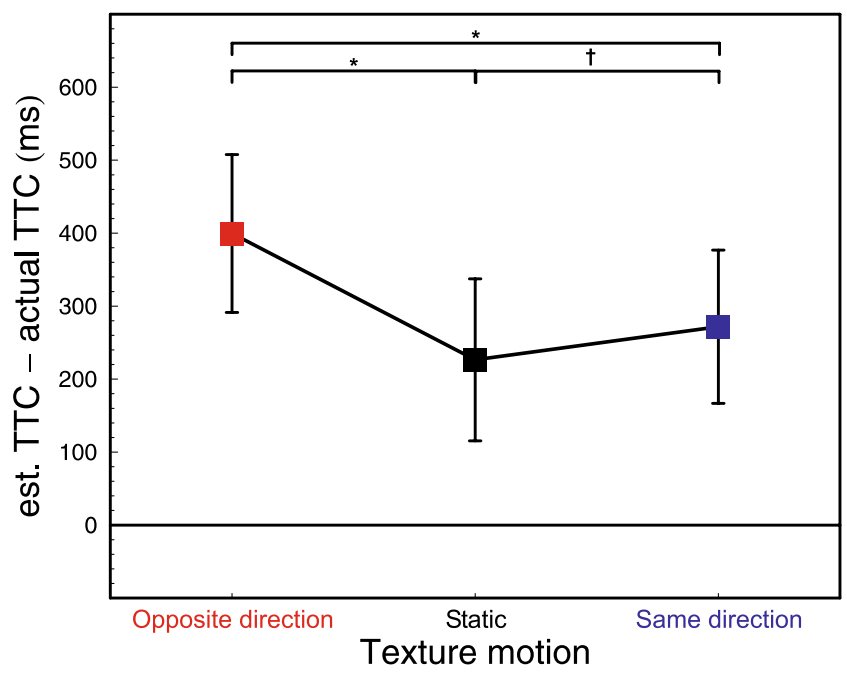

Fig. 6 Experiment 2. Mean constant error as a function of texture motion. The brackets indicate pairwise differences, ${ }^{*} p<.05, \dagger p<.1$. Error bars $95 \%$ CIs increasing TTC. Four post-hoc ANOVAs showed that the effect of texture motion was significant at all TTCs $\left(500 \mathrm{~ms}: F(2,38)=10.9, p<.001, \widetilde{\varepsilon}=.93, \eta^{2}=.37\right.$; $1,000 \mathrm{~ms}: F(2,38)=17.0, p<.001, \widetilde{\varepsilon}=1.0, \eta^{2}=.47$; $1,500 \mathrm{~ms}: F(2,38)=18.1, p<.001, \widetilde{\varepsilon}=1.0, \eta^{2}=.49$; 2,000 ms: $\left.F(2,38)=21.5, p<.001, \widetilde{\varepsilon}=1.0, \eta^{2}=.93\right)$.

None of the remaining main and interaction effects in the main ANOVA was significant (all $p$ values $>.15$ ).

Because there was no significant effect of velocity on the constant error, the variable error (VE; cf. Hartmann, 1983; Tresilian, 1994) for each observer and each Texture Motion $\times$ TTC $\times$ Presentation Duration combination was computed as the standard deviation of the CEs across the seven different velocities. A repeated-measures ANOVA with the within-subject effects texture motion, TTC, and presentation duration on the $\mathrm{VE}$ showed a significant effect of TTC, $F(3,57)=46.8, p<.001, \widetilde{\varepsilon}=.67, \eta^{2}=.71$. As Fig. $7 \mathrm{~b}$ shows, the VE increased with increasing TTC, compatible with previous findings in PM tasks (DeLucia \& Liddell, 1998; Oberfeld \& Hecht, 2008; Schiff \& Detwiler, 1979). The Texture Motion $\times$ TTC interaction was also significant, $F(6,114)=2.75, p=.027, \widetilde{\varepsilon}=.76, \eta^{2}=.13$, with a slightly stronger increase in VE with TTC for the samedirection texture motion. However, the main effect of texture motion on the VE as well as all remaining effects were not significant (all $p$ values $>.05$ ).

Although the dependence of the TTC estimates on the experimental parameters differed from the pattern observed in Experiment 1, most likely owing to the difference between TTC judgments for "looming" objects versus objects moving in the frontoparallel plane, the effects of the task-irrelevant texture motion confirmed the results from Experiment 1. Texture motion in the opposite direction than the contour motion resulted in a TTC overestimation, relative to the static condition. However, texture motion in the same direction as the contour motion did not have a comparable effect, it even caused a slight relative overestimation. Thus, as in Experiment 1, texture motion "amplifying" the global motion fails to elicit earlier TTC estimates. This asymmetry indicates a difference between the effects of ecologically plausible task-irrelevant motion on the one hand and taskrelevant texture motion on the other hand, which is ecologically implausible.

\section{Experiment 3: effects of task-relevant texture motion in a prediction-motion paradigm}

Experiments 1 and 2 provided evidence against a simple integration of global and local, task-irrelevant motion signals in the sense of a weighted average. This pattern observed with task-irrelevant local motion differs from the results obtained when both global and local motion are 


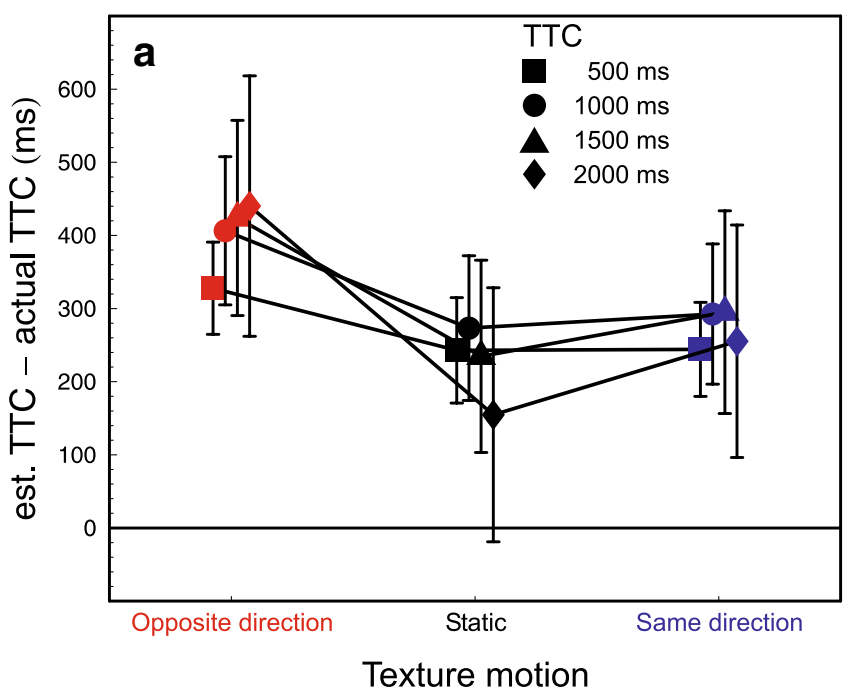

Fig. 7 Experiment 2. a Mean constant error as a function of texture motion and TTC. Boxes, circles, triangles, and diamonds correspond to TTCs of $500,1,000,1,500$, and 2,000 ms, respectively. b Mean variable error as a function of texture motion and TTC. Boxes, circles,

task-relevant, such as in the experiment by Vincent and Regan (1997). Before concluding that the information processing of the visual system differs between taskrelevant and task-irrelevant local motion, however, it is necessary to rule out a potential procedural effect. In the experiments by Vincent and Regan (1997) and Harris and Giachritsis (2000), observers decided on each trial whether the approaching object would have arrived at their position earlier or later than a tone. The temporal position of this tone was varied by an adaptive procedure so that the onset of the tone matched the perceived TTC of the object. In contrast, in the experiments reported in the present study, the task was to press a button at exactly the instant when the object would have arrived at the observer's position or at the finish line. While both tasks obviously require a TTC judgment, the difference between the two procedures might have caused the different pattern of results observed in our experiments as compared to the experiment by Vincent and Regan (1997). To rule out this possibility, we presented the same type of stimuli as used by Vincent and Regan (1997) in a prediction-motion task. A square approaching the observer on the mid-saggital plane was textured with a checkerboard-like pattern. The texture elements could expand either at the same rate as the contour, or at a slower rate, or at a faster rate, or they remained constant in size. If the use of a PM procedure were the cause of the general tendency towards relative overestimation observed in our Experiments 1 and 2, then both the slower-expanding and the faster-expanding texture elements should cause an overestimation relative to the condition where the local rate of expansion matched the global rate of expansion. If,

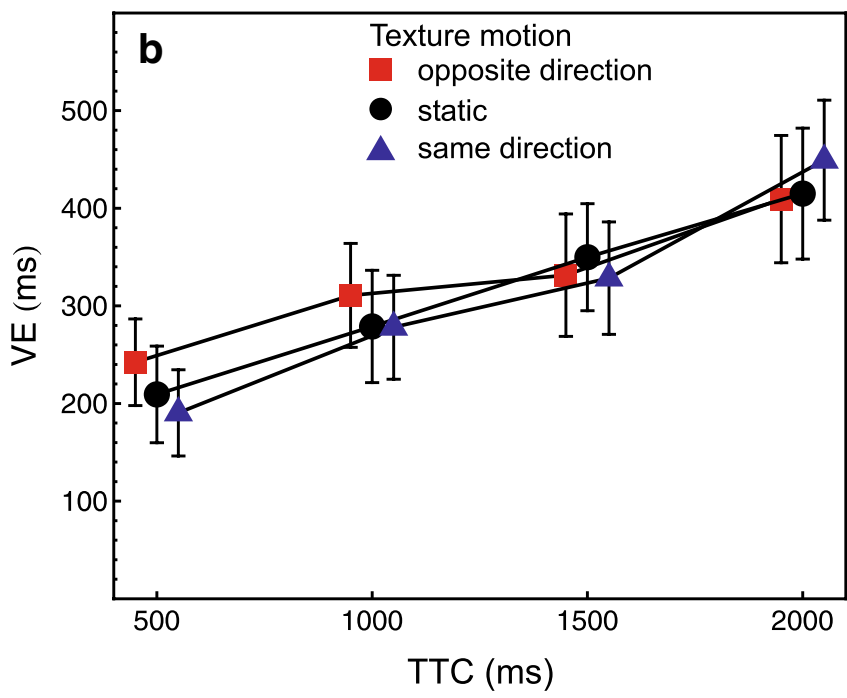

and triangle, correspond to texture motion in the opposite direction as the contour, the static condition, and texture motion in the same direction as the contour, respectively. Error bars $95 \%$ CIs

however, the difference between our results and the findings by Vincent and Regan (1997) are not owed to a mere procedural effect but due to the difference between task-relevant and task-irrelevant local motion, then we should observe a similar pattern as reported by Vincent and Regan (1997). More specifically, for the texture elements expanding at a faster rate than the contour, an underestimation of TTC should be observed relative to the condition where the global and the local expansion rates are identical.

\section{Method}

\section{Observers}

Fifteen volunteers ( 6 women, 9 men) participated in the experiment for partial course credit or payment $(€ 5 / h)$. The participants ranged in age from 19 to 36 years (mean 24.5, SD 4.0). All had normal or corrected-to-normal vision. All participants gave written informed consent according to the Declaration of Helsinki after the course of the experiment, the motivation of the study, and potential risks had been explained to them. They were uninformed about the hypotheses under test.

\section{Stimuli and apparatus}

The same apparatus as in Experiments 1 and 2 was used.

The observers were tested individually in a dimly lit room watching the CRT display binocularly from a distance of $80 \mathrm{~cm}$. The display subtended $22.1^{\circ}$ of visual angle 
horizontally and $16.6^{\circ}$ vertically. A chinrest was used to align the line of sight with the center of the display.

Figure 8 shows a schematic depiction of the stimuli. A textured square was presented with different ratios $(R)$ between the expansion rate of the texture elements and the expansion rate of the contour. To create this type of stimuli, two animated objects were presented. The first object (termed frame in the following) was a large gray opaque square with a square opening (i.e., a "window" with an aperture of $2 \mathrm{~m} \times 2 \mathrm{~m}$ ) and oriented orthogonally to the line of sight, like a big picture frame. The frame always filled the screen completely, thus its outer edges were never visible. The frame moved towards the observer at constant speed on a collision course, horizontally centered on the screen. For an observer fixating the midpoint of the frame, it moved on the mid-saggital plane. The second object (termed textured object in the following) was a large square filled with the same type of black-and-white checkerboardlike pattern (see Fig. 8) as used by Vincent and Regan (1997). The textured object approached the observer with the same velocity as the frame, on the same trajectory, and oriented in parallel to the frame, but it was positioned $2 \mathrm{~mm}$ farther away from the observer than the frame, so that the frame was always in front of the textured object and thus the textured object was visible only in the opening of the frame. The textured object always filled the complete opening of the frame.

\section{Procedure and design}

At $t=0$, both objects appeared on the screen and instantly started to move towards the observer with constant velocity $v_{\mathrm{f}}(20.8,25.0$, or $29.2 \mathrm{~m} / \mathrm{s})$. Both objects disappeared from the screen at $t_{\mathrm{Blank}}=500,1,250$, or $2,000 \mathrm{~ms}$ after trial onset. These parameter values were selected in order to be compatible with Experiments 1 and 2. Note that in the original experiment by Vincent and Regan (1997), the presentation duration was varied within a smaller range $(0.735-1.37 \mathrm{~s})$. The TTC for the frame (i.e., the time between $t_{\text {Blank }}$ and the instant at which the frame would have reached the observer), was 500, 1,000, 1,500, or $2,000 \mathrm{~ms}$. For each velocity, the distance of the frame from the observer at time $t=0, d_{\mathrm{f}}(0)$, was selected to produce the designated value of $t_{\text {Blank }}+$ TTC, which resulted in starting distances between 21 and $117 \mathrm{~m}$. To reduce the correlation between critical optical variables (see above), one of two different scaling factors $(s f=0.8$ or 1.25$)$ was applied to both objects on a given trial, resulting in effective frame sizes of $s=1.6$ and $2.5 \mathrm{~m}$. As a result, none of the optical variables initial retinal size, final retinal size, the rate of retinal angular change at $t=0$ or $t=t_{\mathrm{blank}}$, or the change in visual angle across the trial provided an unambiguous cue to TTC.

The critical independent variable for this experiment was the ratio $R$ between the expansion rate of the texture elements and the expansion rate of the contour (the frame). To realize expansion rates of the texture elements smaller than $(R<1)$, equal to $(R=1)$, and greater than $(R>1)$ the expansion rate of the contour, the size of the textured object was scaled with a time-varying scaling factor scale $(t)$ at each instant $t$. Similar as in the experiment by Vincent and Regan (1997), $R$ was set to $0,0.5,0.8,1.0,1.25$, and 2.0 (see Fig. 8). Note that $R=0$ corresponds to a condition where the size of the texture elements remains constant across time. For each combination of presentation duration, TTC of the frame, and frame velocity, scale $(t)$ was so chosen that the scaling factor was unity when the objects disappeared from the screen, i.e., $\operatorname{scale}\left(t_{\mathrm{blank}}\right)=1$. Therefore, for a given approach of the frame
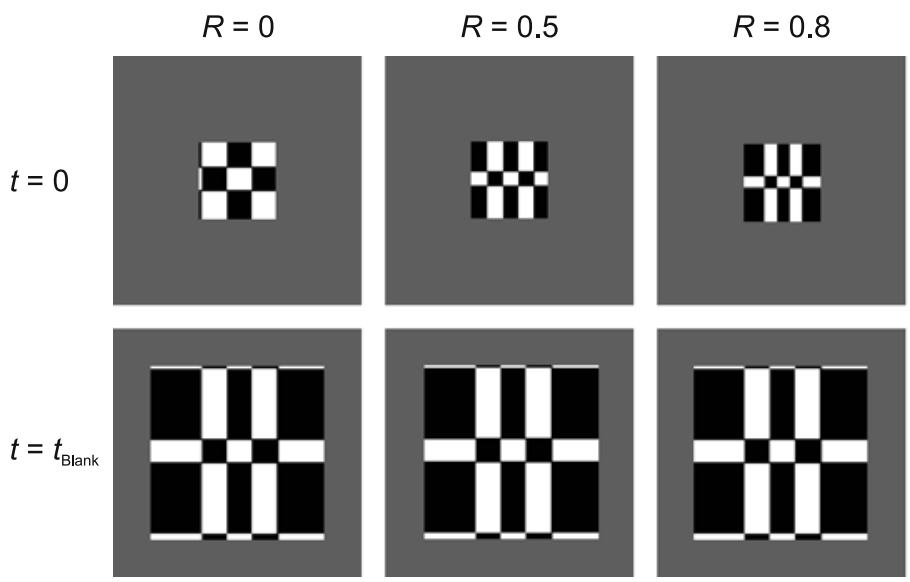
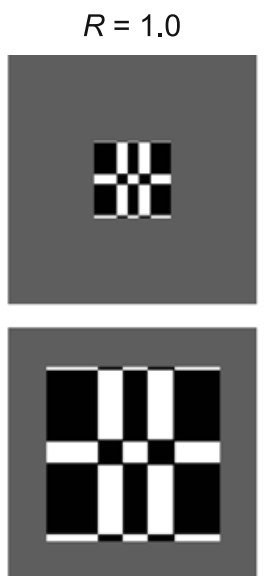
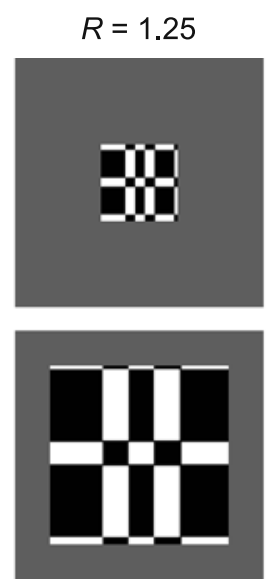
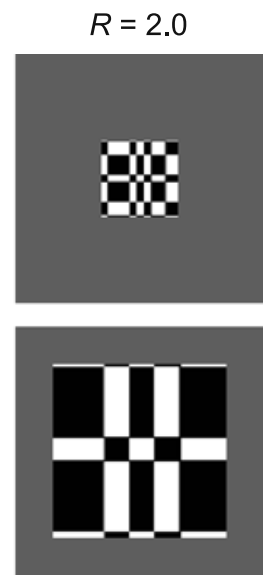

Fig. 8 Experiment 3. Screenshots of different stimulus displays. Each column shows a different value of $R$, the ratio between the expansion rate of the texture elements and the expansion rate of the contour. Upper row initial video frame $\left(t=t_{0}\right)$. Lower row final video frame $\left(t=t_{\mathrm{Blank}}\right)$.
All columns are for $t_{\text {Blank }}=1,250 \mathrm{~ms}$, TTC $=1,000 \mathrm{~ms}, v_{\mathrm{f}}=25.0 \mathrm{~ms}^{-1}$, and the higher scaling factor. Note that the final size of the texture elements was independent of $R$ (see text) 
$\left(t_{\text {Blank }} \times \mathrm{TTC} \times v_{\mathrm{f}} \times s f\right)$, the final size of the texture elements was identical for each value of $R$ (cf. Fig. 8). This is an important difference to the original experiment by Vincent and Regan (1997) where the final size of the texture elements increased with increases in $R$ (see their Fig. 1). As a consequence, if a participant had used the final size as a cue when estimating TTC (e.g., DeLucia, 1991; DeLucia, et al., 2003), then he or she would have produced smaller TTC estimates at higher values of $R$, and longer estimates at lower values of $R$, which is indeed what the data by Vincent and Regan (1997) showed. Our design avoided this potential confound between the ratio of expansion rates and final texture element size.

For $R=1, \operatorname{scale}(t)=1$ for all $t$, and thus the rate of expansion of an imaginary texture element corresponding to the same viewing angle as the frame is identical to the rate of expansion of the frame at each $t$. In other words, for $R=1$, the observers simply viewed a rigid textured square approaching them. For $R \neq 1$, the scaling factor scale $(t)$ was so chosen that at each instant $t$ the rate of expansion of an imaginary texture element corresponding to the same viewing angle as the frame was $R$ times the expansion rate of the frame at the same instant. If the scaling factor increased with $t$, then the texture expansion rate was higher than the contour expansion rate $(R>1)$. If the scaling factor decreased with $t$, then $R<1$. In either condition, we chose $\operatorname{scale}\left(t_{\text {blank }}\right)=1$ to decouple the final texture element size from $R$.

The independent variables $t_{\text {blank }}$, TTC, $v_{\mathrm{f}}, s f$, and $R$ were combined factorially, and each of the $3 \times 4 \times 3 \times 2 \times 6=$ 432 combinations was presented once in each of the two experimental sessions, resulting in a total of two trials per combination. The presentation order was randomized.

The observer pressed a response button with the index finger of the dominant hand at the instant at which he or she thought that the object would have arrived at his or her position. The next trial started automatically after a pause of $2 \mathrm{~s}$ during which the screen was uniformly gray.

The experiment started with a practice block containing 70 trials drawn at random without replacement from the set of 432 conditions. Visual trial-by-trial feedback was provided in form of the signed deviation in milliseconds of the time of the observer's key-press from the actual target TTC. The observers were informed that the initial and final size of the object, its speed, and the presentation duration would be varied and that none of these variables provided a reliable cue to contact time. The observers were instructed to judge only the contour motion and to ignore the texture motion.

In the main part of the experiment, no feedback was provided. Each observer received each of the 432 combinations once in each of the two sessions. Each experimental session lasted approximately $60 \mathrm{~min}$, including two short breaks.

\section{Results and discussion}

Inspection of the data showed that, for one participant, the correlation between the TTC and the response time was not significant in the condition in which the local and the global expansion rate were identical $(R=1)$. This participant appeared to have always pressed the response button at a constant delay after the disappearance of the object from the screen rather than producing TTC estimates. Therefore, the data from this participant were excluded from all further analyses.

For the remaining 14 participants, the constant error was analyzed via a six-factorial repeated-measures ANOVA with Huynh-Feldt $d f$-correction, displayed in Table 2. The within-subjects factors were $R$, presentation duration, TTC, frame velocity, scaling factor, and session. There was a significant effect of $R$ on the CE. As can be seen in Fig. 9, conditions with texture expansion rates smaller than the global expansion rates resulted in a relative overestimation of TTC relative to the condition where the two expansion rates were consistent $(R=1)$. For texture expansion rates higher than the global expansion rate $(R>1)$, we observed a small relative TTC underestimation. Post-hoc pairwise comparisons between the $\mathrm{CE}$ at $R=1$ and the $\mathrm{CE}$ at the other levels of $R$ were computed using non-pooled error terms and Hochberg's (1988) procedure. Only the CE at $R=$ 2 did not differ significantly from the $\mathrm{CE}$ at $R=1$. Thus, we observed the same pattern as Vincent and Regan (1997).
Table 2 Experiment 3: results of the repeated-measures ANOVA on the constant error. Only effects mentioned in the text are displayed. Same format as Table 1

\begin{tabular}{lllllll}
\hline Factor & Num. $d f$ & Den. $d f$ & $F$ & $p$ & $\widetilde{\varepsilon}$ & Partial $\eta^{2}$ \\
\hline $\boldsymbol{R}$ & $\mathbf{5}$ & $\mathbf{6 5}$ & $\mathbf{1 9 . 3 0}$ & $<.001$ & $\mathbf{. 6 0}$ & $\mathbf{. 5 9 8}$ \\
$\boldsymbol{R} \times$ TTC & $\mathbf{1 5}$ & $\mathbf{1 9 5}$ & $\mathbf{2 . 5 1}$ & $\mathbf{. 0 0 5}$ & $\mathbf{. 7 9}$ & $\mathbf{. 1 6 2}$ \\
TTC & $\mathbf{3}$ & $\mathbf{3 9}$ & $\mathbf{2 5 . 4 2}$ & $<.001$ & $\mathbf{. 3 5}$ & $\mathbf{. 6 6 2}$ \\
Presentation duration $\left(t_{\text {blank }}\right)$ & 2 & 26 & 3.44 & .062 & .78 & .209 \\
Velocity $\left(\boldsymbol{v}_{\mathbf{f}}\right)$ & $\mathbf{2}$ & $\mathbf{2 6}$ & $\mathbf{3 8 . 7 7}$ & $<.001$ & $\mathbf{. 7 6}$ & $\mathbf{. 7 4 9}$ \\
Scaling factor $(s \boldsymbol{f})$ & $\mathbf{1}$ & $\mathbf{1 3}$ & $\mathbf{3 5 . 5 1}$ & $<.001$ & $\mathbf{1 . 0 0}$ & $\mathbf{. 7 3 2}$ \\
TTC $\times \boldsymbol{t}_{\text {blank }}$ & $\mathbf{6}$ & $\mathbf{7 8}$ & $\mathbf{6 . 6 9}$ & $<.001$ & $\mathbf{. 7 4}$ & $\mathbf{. 3 4 0}$ \\
\hline
\end{tabular}




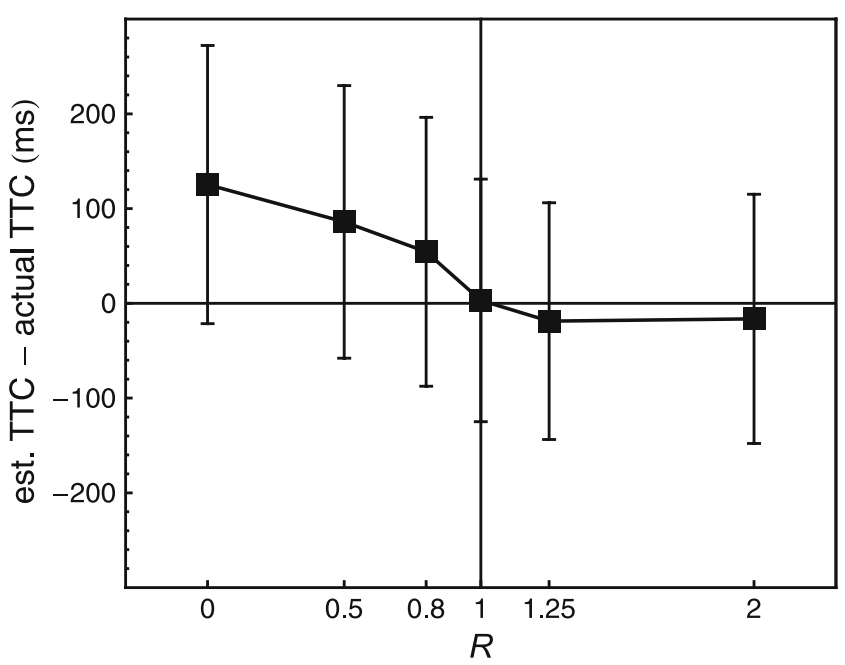

Fig. 9 Experiment 3. Mean constant error as a function of $R$, the ratio of texture expansion rate and contour expansion rate. Error bars 95\% CIs

However, on average, the maximum change in the $\mathrm{CE}$ caused by values of $R$ differing from unity was only $122 \mathrm{~ms}$ in our experiment, while Vincent and Regan (1997) reported a mean change of more than $500 \mathrm{~ms}$ for their two participants (see their Fig. 2).

The ANOVA (Table 2) also showed a significant $R \times$ TTC interaction. As Fig. 10 shows, the effect of $R$ was more pronounced at longer TTCs, compatible to the effect observed in Experiment 1. The remaining interactions involving $R$ were not significant (all $p$ values $>.05$ ).

As in Experiment 1, the CE decreased significantly with TTC, compatible with previous studies (e.g., Oberfeld \& Hecht, 2008). The CE also showed a non-significant trend to decrease with $t_{\text {blank, }}$, just as in Experiment 1 . There was a significant TTC $\times t_{\text {blank }}$ interaction, most likely due to a smaller effect of TTC at the shortest $t_{\text {blank. The CE was }}$ significantly smaller for the greater scaling factor, and increased significantly with $v_{\mathrm{f}}$. Both effects are compatible with the use of the pictorial cue 'final size' (see Results section for Experiment 1). Taken together, the effects of the motion parameters show the pattern typically observed in PM experiments.

To summarize the results of Experiment 3, which used the same type of texture motion as Vincent and Regan (1997), we found the same pattern of effects on the TTC estimates in a prediction motion task as observed by Vincent and Regan (1997) using a different task. Thus, it is the specific texture motion rather than the use of the PM task that caused the different effects in Experiment 3 as opposed to Experiments 1 and 2. With the task-relevant texture motion in Experiment 3, we observed integration of global and local motion in the sense of a weighted average. With the task-irrelevant texture motion in Experiments 1 and 2 , on the other hand, we observed a different pattern. It should be noted, that while qualitatively similar, the effects of $R$ on the TTC estimates observed in Experiment 3 were much smaller than in the original study by Vincent and Regan (1997). This difference in results is likely due to the different selection of experimental parameters. Most importantly, in the study by Vincent and Regan (1997), the final size of the texture elements was correlated with $R$. Our experiments carefully avoided this confound, so that for our data the effects of $R$ on the TTC estimates cannot be explained by the use of the pictorial cue final size. Finally, our tests of potential procedural influences is incomplete in the sense that it would be interesting to use the "tone task" by Vincent and Regan (1997) combined with our taskirrelevant texture motions from Experiments 1 and 2.

\section{General discussion}

We explored the role of task-irrelevant local motion on TTC estimation and found that local motion does interfere. The stimuli corresponded to possible approaches of real, rigid objects rather than being physically impossible, and they avoided pictorial cues that had been problematic in previous studies (e.g., Vincent \& Regan, 1997). In Experiment 1, we presented stimuli on sagittal approach trajectories that contained local motion (illusory expanding or contracting textures). This local motion was taskirrelevant because it did not provide information about TTC. In Experiment 2, we chose frontoparallel motion to find out if interference of local texture motion is limited to radial expansion patterns. In both experiments, we found consistent effects of the task-irrelevant texture motion suggesting that local texture motion has the general ability

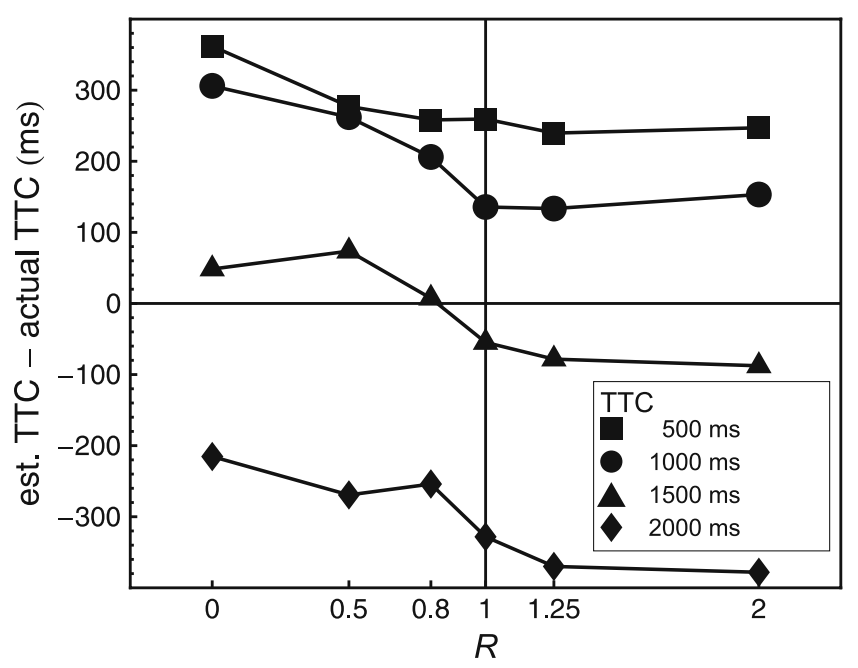

Fig. 10 Experiment 3. Mean constant error as a function of $R$ and TTC. Boxes, circles, triangles, and diamonds correspond to TTCs of $500,1,000,1,500$, and 2,000 ms, respectively 
to interfere with global motion processing. We also found an asymmetry of interference. Texture motion "working against" global motion (e.g., the illusory contracting spiral in Experiment 1) caused an overestimation of TTC, compared to the static condition without texture motion. However, texture motion that should have strengthened the global motion (e.g., illusory expansion in Experiment 1) did not result in a relative TTC underestimation. This asymmetry rules out a simple averaging of local and global motion. Interestingly, these effects differ from the effects of local motion reported in previous studies (Harris \& Giachritsis, 2000; Vincent \& Regan, 1997). We suggest that, for the mismatch type of stimuli presented in the latter studies, local motion was perceived as task-relevant because in our natural environment the expansion rate of texture elements is strongly correlated with the TTC of the object. Our third experiment replicated the task-relevant local motion used by Vincent and Regan (1997) but at the same time eliminated alternative cues that might have guided TTC judgments. In this replication, the effects of local motion were compatible with integration (averaging) of the local and the global motion signals. Thus, our distinction between task-relevant and task-irrelevant local motion was justified. The pattern of effects caused by our task-irrelevant texture motion were robust in the sense that we found them for two different planes of motion, and more importantly for two rather different types of texture motion, one illusory and one non-illusory.

Our results suggest the involvement of two different processes. The first process would be an integration of contour motion and texture motion, in the sense of a weighted average. The second process could be described as a general slowing of the response due to the taskirrelevant texture motion, which could for instance be due to a capacity-limited processing stage. Process 1 would explain why the change in the TTC estimates differed between the two different directions of texture motion (e.g., in the same direction as the contour motion versus in the opposite direction than the contour motion). Process 2, on the other hand, would explain the general tendency towards TTC overestimation, which was found even with expanding texture (Experiment 1) or the stripes moving in the same direction as the contour (Experiment 2). Note that DeLucia and Novak (1997) reported evidence for limited-capacity processing when judging the relative time-to-contact of more than two approaching objects (see also Andersen \& Kim, 2001), although they looked at accuracy rather than at systematic shifts towards over- or underestimation. These results might indicate that dividing attention between several sources of motion information affects performance. It should be noted, however, that the presence of additional moving and task-irrelevant display elements does not in all cases result in TTC overestimation. Apparently, the task- irrelevant elements need to belong to the object whose TTC is to be judged. Oberfeld and Hecht (2008) found that a separate moving distractor object caused a relative TTC underestimation of a target object in most conditions. That is, a diverging pattern arose when two spatially distinct objects (target and distractor) were presented, while in the experiments reported here the task-irrelevant motion was located within the target object.

For the rotating spiral presented in Experiment 1, it could be argued that the non-significant effect of the illusory expansion was due to an asymmetry in the effectiveness of a spiral's rotation to induce motion in depth. As noted by Reinhardt-Rutland (1994, p. 767, word order changed), "[...] the lack of some properties of real motion of objects in depth renders spiral recession more convincing than spiral approach." However, Experiment 2 presented a completely different, non-illusory type of local motion, and the same effects of task-irrelevant texture motion were observed. In more general terms, there have been reports from other paradigms indicating that it is easier to attenuate than to amplify motion signals. Linares, López-Moliner, and Johnston (2007) reported that adding an extra motion signal to a flash-lag experiment (e.g., Nijhawan, 1994) in the form of a motion after-effect reduced the flash-lag effect if the direction of the aftereffect was against the object motion. If the direction of the motion after-effect was the same as the object motion, however, it did not modulate the flash-lag effect. Note also that a similar asymmetry was present in the data by Vincent and Regan (1997), where local expansion rates that were slower than the global expansion rate had a stronger effect than local expansion rates that exceeded the global expansion rate.

Texture motion could also direct the attention, or the actual fixation of the subjects, to different parts of the stimulus than in a condition without texture motion. Eye movements can have an effect on TTC estimation (e.g., Bennett, Baures, Hecht, \& Benguigui, 2010). However, we currently do not have a clear hypothesis about how eye movements or spatial selective attention induced by texture motion could cause the specific pattern of results were observed.

The distractor paradigm as developed in our present work has the potential to further elucidate mechanisms of motion processing. Models of looming perception typically assume isotropic expansion that can be registered by pairs of filters with opposing directional preferences (e.g., Beverley \& Regan, 1980; van de Grind, 1990). Added local, radial motion can still be handled by nested sets of filter pairs, although at the cost of a compromise ("weighted average"), as suggested by Gray and Regan (1999). While a rotational component has often been shown to be neutral (e.g., Freeman \& Harris, 1992; but see Simpson, 1988), local translatory motion, as it was present in our stimuli, seems to 
impair extraction of the global radial flow. Single and multiple drifting gratings ("plaids"; cf. van den Berg \& van de Grind, 1991) could be used to further investigate the susceptibility of looming-sensitive mechanisms to local perturbations.

As noted above, we used rather artificial and simple laboratory stimuli. So do our findings have implications for the real world? As one reviewer noted, one example of task-irrelevant "texture motion" would be a passenger moving inside an approaching vehicle. Another example becoming more and more frequent would be a modern bus with an information or advertizing display that contains moving stimuli. From a viewpoint of traffic safety, it might be worthwhile studying whether such dynamic displays on vehicles produce a tendency towards TTC overestimation, just as did the texture motion in our stimuli.

Taken together, our experiments show that local texture motion in ecologically plausible contexts does interfere with TTC estimation in a rather specific way. The taskirrelevant motion cannot be ignored. It is factored into the estimate, and it seems to slow down the response.

Acknowledgments This work was supported by a grant from Deutsche Forschungsgemeinschaft (DFG) to Heiko Hecht (HE 2122/ 6-1: Kontaktzeitschätzung im Kontext). We are grateful to Agnes Münch for her programming expertise. Franziska Baldauf, Elsa Krauss and Felicitas Klöckner helped with table and figure preparation. We thank three anonymous reviewers for helpful comments on an earlier version of this paper.

\section{References}

Andersen, G. J., \& Kim, R. D. (2001). Perceptual information and attentional constraints in visual search of collision events. Journal of Experimental Psychology: Human Perception and Performance, 27(5), 1039-1056.

Bennett, S., Baures, R., Hecht, H., \& Benguigui, N. (2010). Eye movements influence estimation of time-to-contact in prediction motion. Experimental Brain Research, 1-9. doi:10.1007/s00221010-2416-y

Beverley, K. I., \& Regan, D. (1980). Visual sensitivity to the shape and size of a moving object: Implications for models of object perception. Perception, 9(2), 151-160.

Beverley, K. I., \& Regan, D. (1983). Texture changes versus size changes as stimuli for motion in depth. Vision Research, 23(12), 1387-1399. doi:0042-6989(83)90150-5 [pii]

Bootsma, R. J., \& Craig, C. M. (2002). Global and local contributions to the optical specification of time to contact: Observer sensitivity to composite tau. Perception, 31(8), 901-924. doi:10.1068/p3230

DeLucia, P. R. (1991). Pictorial and Motion-Based Information for Depth-Perception. Journal of Experimental Psychology: Human Perception and Performance, 17(3), 738-748.

DeLucia, P. R., Kaiser, M. K., Bush, J. M., Meyer, L. E., \& Sweet, B. T. (2003). Information integration in judgements of time to contact. The Quarterly Journal of Experimental Psychology. A: Human Experimental Psychology, 56(7), 1165-1189. doi:10.1080/02724980343000053
DeLucia, P. R., \& Liddell, G. W. (1998). Cognitive motion extrapolation and cognitive clocking in prediction motion tasks. Journal of Experimental Psychology: Human Perception and Performance, 24(3), 901-914.

DeLucia, P. R., \& Novak, J. B. (1997). Judgments of relative time-tocontact of more than two approaching objects: Toward a method. Perception \& Psychophysics, 59(6), 913-928.

DeLucia, P. R., Tresilian, J. R., \& Meyer, L. E. (2000). Geometrical illusions can affect time-to-contact estimation and mimed prehension. Journal of Experimental Psychology: Human Perception and Performance, 26(2), 552-567.

Freeman, T. C. A., \& Harris, M. G. (1992). Human sensitivity to expanding and rotating motion - effects of $=$ complementary masking and directional structure. Vision Research, 32(1), 81-87.

Giachritsis, C. D., \& Harris, M. G. (2005). Global versus local image expansion in estimating time-to-contact from complex optic flow. Perception, 34(5), 577585.

Gray, R., \& Regan, D. (1998). Accuracy of estimating time to collision using binocular and monocular information. Vision Research, 38(4), 499-512.

Gray, R., \& Regan, D. (1999). Motion in depth: Adequate and inadequate simulation. Perception \& Psychophysics, 61(2), 236-245.

Gray, R., \& Regan, D. (2000). Estimating the time to collision with a rotating nonspherical object. Vision Research, 40(1), 49-63.

Harris, M. G., \& Giachritsis, C. D. (2000). Coarse-grained information dominates fine-grained information in judgments of time-tocontact from retinal flow. Vision Research, 40(6), 601-611. doi:10.1016/S0042-6989(99)00209-6

Hartmann, W. M. (1983). Localization of sound in rooms. The Journal of the Acoustical Society of America, 74(5), 1380-1391.

Hecht, H., Kaiser, M. K., Savelsbergh, G. J. P., \& van der Kamp, J. (2002). The impact of spatiotemporal sampling on time-tocontact judgments. Perception \& Psychophysics, 64(4), 650-666.

Hecht, H., \& Savelsbergh, G. J. P. (Eds.). (2004). Time-to-contact. Amsterdam: Elsevier.

Heuer, H. (1993). Estimates of time to contact based on changing size and changing target vergence. Perception, 22(5), 549-563.

Hochberg, Y. (1988). A sharper Bonferroni procedure for multiple tests of significance. Biometrika, 75(4), 800-802.

Holland, H. C. (1965). The spiral after-effect. Oxford, New York: Pergamon Press.

Hoyle, F. (1957). The black cloud. London: Heinemann.

Huynh, H., \& Feldt, L. S. (1976). Estimation of the Box correction for degrees of freedom from sample data in randomized block and split-plot designs. Journal of Educational Statistics, 1(1), 69-82. doi:http://dx..org/10.2307/1164736

Kaiser, M. K., \& Hecht, H. (1994). Time-to-passage judgments under violations of various constancy assumptions. Paper presented at the 35th Annual Meeting of the Psychonomic Society.

Kaiser, M. K., \& Mowafy, L. (1993). Optical specification of time-topassage - observers sensitivity to global tau. Journal of Experimental Psychology: Human Perception and Performance, 19(5), 1028-1040.

Kerzel, D., Hecht, H., \& Kim, N. G. (1999). Image velocity, not tau, explains arrival-time judgments from global optical flow. Journal of Experimental Psychology: Human Perception and Performance, 25(6), 1540-1555.

Keselman, H. J. (1994). Stepwise and simultaneous multiple comparison procedures of repeated-measures means. Journal of Educational Statistics, 19(2), 127-162.

Lee, D. N. (1974). Visual information during locomotion. In R. B. McLeod \& H. L. Pick (Eds.), Perception: Essays in honor of James J. Gibson (pp. 250-267). Ithaca: Cornell University Press.

Lee, D. N. (1976). Theory of visual control of braking based on information about time-to-collision. Perception, 5(4), 437-459. 
Linares, D., López-Moliner, J., \& Johnston, A. (2007). Motion signal and the perceived positions of moving objects. Journal of Vision, 7(7), 7. doi:110.1167/7.7.1

López-Moliner, J., Brenner, E., \& Smeets, J. B. J. (2007). Effects of texture and shape on perceived time to passage: Knowing "'what" influences judging "when". Perception \& Psychophysics, 69(6), 887-894.

McLeod, R. W., \& Ross, H. E. (1983). Optic flow and cognitive factors in time-to-collision estimates. Perception, 12(4), 417423.

Morrone, M. C., Burr, D. C., \& Vaina, L. M. (1995). Two stages of visual processing for radial and circular motion. Nature, 376 (6540), 507-509.

Nijhawan, R. (1994). Motion extrapolation in catching. Nature, 370 (6487), 256-257.

Oberfeld, D., \& Hecht, H. (2008). Effects of a moving distractor object on time-to-contact judgments. Journal of Experimental Psychology: Human Perception and Performance, 34(3), 605623. doi:10.1037/0096-1523.34.3.605

Plateau, J. (1850). Vierte Notiz über neue, sonderbare Anwendungen des Verweilens der Eindrücke auf die Netzhaut [Fourth note on an unusual application of retinal persistence]. Poggendorff's Annalen der Physik und Chemie, 80, 287-292.

Purdy, W. C. (1958). The hypothesis of psychophysical correspondence in space perception. Dissertation Abstracts International, $42,1454$.

Reinhardt-Rutland, A. H. (1994). Perception of motion in-depth from luminous rotating spirals - directional asymmetries during and after rotation. Perception, 23(7), 763-769.

Savelsbergh, G. J. P., Whiting, H. T. A., \& Bootsma, R. J. (1991). Grasping Tau. Journal of Experimental Psychology: Human Perception and Performance, 17(2), 315-322.
Schiff, W., \& Detwiler, M. L. (1979). Information used in judging impending collision. Perception, 8(6), 647-658.

Schiff, W., \& Oldak, R. (1990). Accuracy of judging time to arrival effects of modality, trajectory, and gender. Journal of Experimental Psychology: Human Perception and Performance, 16(2), 303-316.

Scott, T. R., \& Noland, J. H. (1965). Some stimulus dimensions of rotating spirals. Psychological Review, 72(5), 344-357.

Simpson, W. A. (1988). Depth discrimination from optic flow. Perception, 17(4), 497-512.

Tresilian, J. R. (1990). Perceptual information for the timing of interceptive action. Perception, 19(2), 223-239.

Tresilian, J. R. (1991). Empirical and theoretical issues in the perception of time to contact. Journal of Experimental Psychology: Human Perception and Performance, 17(3), 865-876.

Tresilian, J. R. (1994). Approximate information sources and perceptual variables in interceptive timing. Journal of Experimental Psychology: Human Perception and Performance, 20(1), 154-173.

Tresilian, J. R. (1995). Perceptual and cognitive-processes in time-tocontact estimation - analysis of prediction-motion and relative judgment tasks. Perception \& Psychophysics, 57(2), 231-245.

van de Grind, W. A. (1990). Smart mechanisms for the visual evaluation and control of self-motion. In R. Warren \& A. H. Wertheim (Eds.), Perception and control of self-motion (pp. 357398). Hillsdale, NJ: Erlbaum.

van den Berg, A. V., \& van de Grind, W. A. (1991). Conditions for the detection of coherent motion. Vision Research, 31(6), 1039-1051.

Vincent, A., \& Regan, D. (1997). Judging the time to collision with a simulated textured object: Effect of mismatching rate of expansion of object size and of texture element size. Perception \& Psychophysics, 59(1), 3236. 